\title{
Probing the Two-Exciton Manifold of Light-Harvesting Antenna Complexes Using Femtosecond Four-Wave Mixing
}

\author{
O. Kuihn ${ }^{\dagger}$ and S. Mukamel* \\ Department of Chemistry, University of Rochester, Rochester, New York 14627
}

Received: June $18,1996^{\otimes}$

Two femtosecond four-wave-mixing techniques carried out using the photon echo pulse sequence with positive or negative time delays are shown to provide a direct probe for two-exciton dynamics in light-harvesting antennae of photosynthetic bacteria and green plants. The spectroscopic signatures of intramolecular and interaction-induced (intermolecular) two-exciton states are analyzed using a collective excitonic oscillator representation of the nonlinear optical response.

\section{Introduction}

The recent availability of high-resolution structural data for several light-harvesting pigment-protein complexes had stimulated intensive spectroscopic investigations of these systems. ${ }^{1-3}$ The two complexes most widely studied are the peripheral antenna of purple bacteria (LH2) and the antenna associated with photosystem II of green plants (LHC-II). The $2.5 \AA$ resolution data for the LH2 of Rhodopseudomonas (Rps.) acidophila show 27 bacteriochlorophyll $a$ (BChl $a$ ) monomers arranged into two rings. ${ }^{4}$ The nine weakly coupled BChl $a$ molecules forming the outer ring are responsible for the higher energetic absorption band (B800), while the 18 strongly interacting inner ring pigments give the lower energetic band (B850). Based on this geometry, there exist several calculations of the intermolecular interaction energies, $J_{m n}$. The reported values for the maximum $J_{m n}$ range from $450^{5}$ to $290 \mathrm{~cm}^{-1}{ }^{6}$ This reflects the uncertainty introduced, e.g., by the lack of knowledge about the dielectric properties of the medium surrounding the BChl $a$ molecules in the native antenna (see discussion in ref 5). In the following we will make use of the results of the excitonic calculations of Pullerits and coworkers. ${ }^{5,7}$ The monomer transition energies for the B800 and the B850 BChl $a$ molecules were taken as 800 and $820 \mathrm{~nm}$. These values reproduce the experimental shift between the B800 and B850 absorption maxima which is $950 \mathrm{~cm}^{-1}$ at low temperatures $(19 \mathrm{~K})$ in Rps. acidophila. ${ }^{8}$ The obtained linear absorption spectrum is plotted in Figure 1a. The solid line contains a Lorentzian broadening with a fwhm of $5 \mathrm{~cm}^{-1}$. The dash-dotted line was obtained by convoluting the stick spectrum with a Gaussian of variance $\sigma=85 \mathrm{~cm}^{-1}$.

LHC-II lacks the high symmetry found in LH2. Twelve chlorophyll (Chl) molecules are arranged into two layers located on the upper and the lower side of the membrane, respectively. ${ }^{9}$ The $3.4 \AA$ resolution obtained for this system does not identify the Chls unambiguously. Using physiological arguments, however, Kühlbrandt and co-workers assigned the seven chlorophylls which are closest to the two carotenoids as Chl $a$ and the remaining ones as Chl $b$. Even if we accept these assignments (which are still controversial), there are two possible orientations for the $Q_{y}$ transition dipole moments of each $\mathrm{Chl}$ molecule. This makes the determination of transition energies and coupling strengths rather difficult. Simulation of circular dichroism line shapes resulted in a unique orientation for only

$\dagger$ Present address: Department of Chemical Physics, Lund University, P.O. Box 124, S-22100 Lund, Sweden.

${ }^{\otimes}$ Abstract published in Advance ACS Abstracts, January 1, 1997.
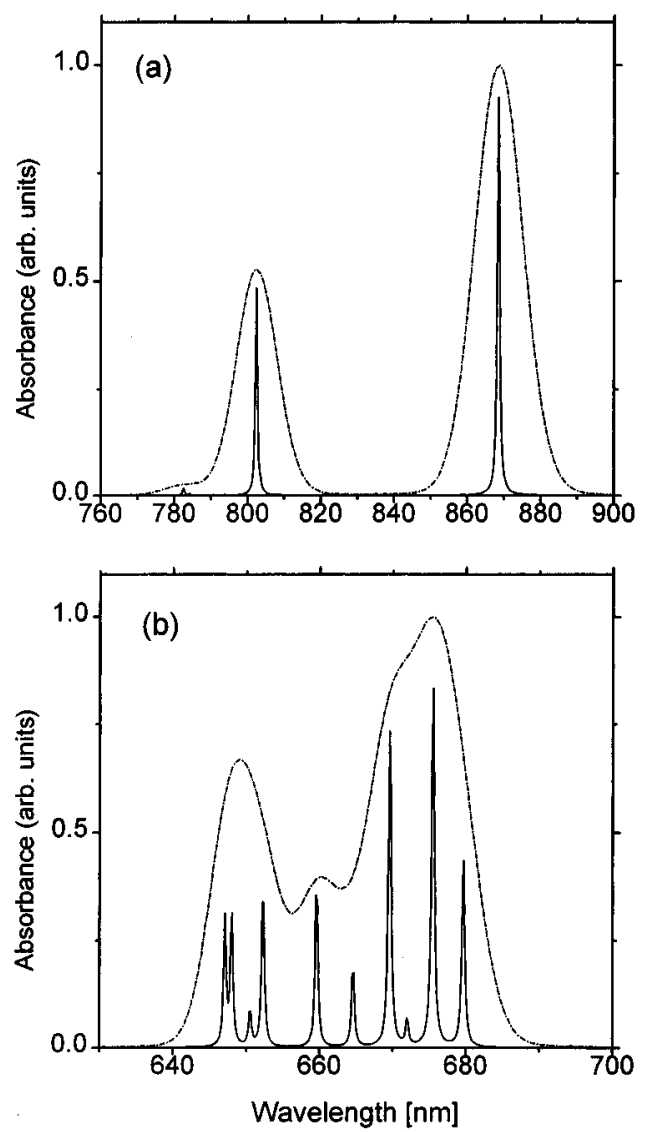

Figure 1. (a) Linear absorption of $\mathrm{LH} 2$ calculated using the parameters of refs 5 and 7. The monomer transitions of the B800 and B850 pigments are at 800 and $820 \mathrm{~nm}$, respectively. Solid line: Stick spectrum with Lorentzian broadening (fwhm: $\Gamma_{n}^{(1)}=5 \mathrm{~cm}^{-1}$ ); dashdotted line: convolution of sticklike spectrum with a Gaussian (variance $\sigma_{n}=85 \mathrm{~cm}^{-1}$ ). (b) Absorption spectrum of LHC-II calculated using the parameters given in ref 12 . The monomer transitions are at (nomenclature as in ref 9): $\Omega_{n=1}(\mathrm{a} 1): 672.7 \mathrm{~nm}, \Omega_{2}(\mathrm{a} 2): 672.7 \mathrm{~nm}$, $\Omega_{3}(\mathrm{a} 3): 667.3 \mathrm{~nm}, \Omega_{4}(\mathrm{a} 4): 672.7 \mathrm{~nm}, \Omega_{5}(\mathrm{a} 5): 678.3 \mathrm{~nm}, \Omega_{6}(\mathrm{a} 6)$ : $664.5 \mathrm{~nm}, \Omega_{7}(\mathrm{a} 7): 659.8 \mathrm{~nm}, \Omega_{8}(\mathrm{~b} 1): 648.1 \mathrm{~nm}, \Omega_{9}(\mathrm{~b} 2): 648.1$ $\mathrm{nm}, \Omega_{10}$ (b3): $652.6 \mathrm{~nm}, \Omega_{11}$ (b5): $652.6 \mathrm{~nm}, \Omega_{12}(\mathrm{~b} 6): 652.6 \mathrm{~nm}$. Broadening as in (a) with $\Gamma_{n}^{(1)}=5 \mathrm{~cm}^{-1}$ and $\sigma_{n}=64 \mathrm{~cm}^{-1}$.

three Chls. ${ }^{10}$ Ultrafast transient absorption data suggest possible orientations for the remaining monomers. ${ }^{11}$ On the other hand, the assumption that the transition dipoles are parallel to the membrane plane was shown to give a reasonable fit to the lowtemperature absorption profile. ${ }^{12}$ This geometry was favored 
to one in which all dipoles are aligned perpendicular to the membrane plane, ${ }^{13}$ since it qualitatively reproduced the transient absorption signal measured in ref 14 . In Figure $1 \mathrm{~b}$ we display the linear absorption spectrum for LHC-II calculated using the parameters of ref 12. The monomer transition energies which followed from the excitonic calculations are given in the figure caption. As in Figure 1a we included a Lorentzian broadening (fwhm $5 \mathrm{~cm}^{-1}$, solid line) and an additional Gaussian broadening with variance $\sigma=64 \mathrm{~cm}^{-1}$ (dash-dotted line).

The progress made in the determination of the molecular structure of antenna complexes has triggered many ultrafast spectroscopic investigations aimed at clarifying the influence of various factors on the energy transfer dynamics. In particular, the $\sim 0.7 \mathrm{ps}$ transfer from the B800 to the B850 band in LH2 at room temperature has been studied thoroughly using one- and two-color pump - probe, ${ }^{15}$ transient grating, and time-integrated two- and three-pulse photon echo setups. ${ }^{16}$ Evidence for the coupling of energy transfer to coherent vibrational motion has also been found. ${ }^{16,17}$ In contrast, the asymmetric nature of LHCII leads to a multitude of energy transfer time scales ranging from several hundred femtoseconds to about $10 \mathrm{ps,} \mathrm{as} \mathrm{demon-}$ strated recently by Vissers and co-workers using the pumpprobe technique. ${ }^{11}$ This study recovered earlier results for subpicosecond transfer times which have been derived from time-resolved fluorescence ${ }^{18,19}$ and pump-probe ${ }^{14,20,21}$ data and extended them over a broad range of pump and probe wavelengths. To date, no signatures of coherent vibrational motion have been reported for LHC-II.

Two-exciton states in aggregates have attracted considerable interest in strongly coupled J-aggregates ${ }^{22}$ and photosynthetic antennae. ${ }^{23}$ There are two types of doubly excited (two-exciton) states in aggregates made of molecules with multiple electronic states. The first, denoted collective double excitations (CDE), represents states in which two molecules are excited to their lowest exciton state. The second type, denoted molecular double excitations (MDE) represents double excitations of individual molecules and exists in the monomer as well. The interplay between these two sources of two-photon absorption has been studied in the frequency domain for linear aggregates ${ }^{24}$ and for aggregates of arbitrary geometry. ${ }^{23}$ When MDE and CDE are energetically close, they may be strongly mixed. We will refer to the entire two-exciton manifold as the two-exciton band. MDE of Chl $a$ in solution shows a broad band around $670 \mathrm{~nm}$ which overlaps with the monomeric ground state absorption band. ${ }^{25}$ Therefore, MDE has been discussed as a source of excited state absorption in time-resolved pump-probe measurements on LHC-II. ${ }^{11,14,26}$ In LH2, MDE of BChl $a$ monomers has been used to explain transient absorption data obtained for Rb. sphaeroides. ${ }^{5}$

The optical response of conjugated molecules ${ }^{27,28}$ and aggregates ${ }^{22,29-32}$ may be adequately described using a coupled excitonic oscillator representation. This picture has several conceptual as well as computational advantages, which will be reviewed briefly in the next section. Using this approach, we have recently shown that the different organization of the pigments in the two antennae is of particular significance for the frequency-domain two-photon resonances evolving from the two-exciton band. ${ }^{23}$ In the present work we extend this study to the time domain and propose two four-wave mixing (FWM) techniques that can directly probe the dynamics within the twoexciton manifold.

In section II we briefly review the excitonic oscillator theory developed in refs 23 and 32 and give the equations of motion for the relevant excitonic variables from which the time-resolved third-order signal is obtained. Numerical results which dem- onstrate the behavior of the two-exciton band in LH2 and LHCII are presented in sections III and IV. In section III we consider a two-pulse FWM technique using the photon echo pulse sequence with negative delay, and in section IV we calculate a two-exciton photon echo which was recently suggested as a new probe for two-exciton dynamics. ${ }^{33}$ Our results are summarized in section $\mathrm{V}$.

\section{Equations of Motion}

We consider an aggregate made of $N$ interacting molecules each described by three electronic states $(|0\rangle,|1\rangle$, and $|2\rangle)$. We assume that only the $|0\rangle \rightarrow|1\rangle$ and the $|1\rangle \rightarrow|2\rangle$ transitions have nonzero (and parallel) transition dipole matrix elements ( $\mu_{n}^{(10)}$ and $\left.\mu_{n}^{(21)}\right)$. We shall denote the frequency of the $|0\rangle \rightarrow|1\rangle$ and the $|1\rangle \rightarrow|2\rangle$ transitions by $\Omega_{n}$ and $\Omega_{n}+\Delta_{n}$, respectively, where $\Delta_{n}$ is an "anharmonicity" parameter. This system is represented by the following Hamiltonian

$$
\begin{array}{r}
H=\sum_{n}\left[\hbar \Omega_{n} B_{n}^{\dagger} B_{n}+\frac{g_{n}}{2}\left(B_{n}^{\dagger}\right)^{2}\left(B_{n}\right)^{2}\right]+\sum_{m n}\left[J_{m n} B_{m}^{\dagger} B_{n}+\right. \\
\left.J_{n m} B_{n}^{\dagger} B_{m}\right]-\sum_{n} \vec{d}_{n} \vec{E}\left(\vec{r}_{n}, t\right)
\end{array}
$$

$B_{n}^{\dagger}\left(B_{n}\right)$ is the creation (annihilation) operator for excitations at the $n$th molecule. These operators satisfy the commutation relations

$$
\left[B_{m}, B_{n}^{\dagger}\right]=\delta_{m n}\left[1+\left(\kappa_{m}{ }^{2}-2\right) B_{m}^{\dagger} B_{\mathrm{m}}\right]
$$

Here, $\kappa_{n}=\mu_{n}^{(21)} / \mu_{n}^{(10)}$ denotes the ratio between the transition dipole moments for $|1\rangle \rightarrow|2\rangle$ and $|0\rangle \rightarrow|1\rangle$ transitions. The parameter $g_{n}$ is related to $\Delta_{n}$ and $\kappa_{n}$ through $^{23}$

$$
g_{n}=2 \hbar\left[\left(2 \Omega_{n}+\Delta_{n} / \hbar\right) \kappa_{n}^{-2}-\Omega_{n}\right]
$$

$J_{m n}$ is the hopping matrix representing dipole-dipole interactions, and the last term in eq 2.1 describes the coupling to the classical external field $\vec{E}(\vec{r}, t)$, where the molecular dipole operator is given by

$$
\vec{d}_{n}=\vec{\mu}_{n}\left(B_{n}+B_{n}^{\dagger}\right)
$$

Here, $\vec{\mu}_{n}$ is the transition dipole matrix element for the $|0\rangle \rightarrow$ |1) transition at the $n$-th molecule. The polarization operator is ${ }^{34}$

$$
P=\sum_{n} \mu_{n}\left(B_{n}+B_{n}^{\dagger}\right)
$$

where the aggregate is assumed to be small compared with the optical wavelength and the vector notation is eliminated for brevity. ${ }^{34}$

This Hamiltonian maps the system onto a set of coupled excitonic oscillators. The oscillator representation has several advantages over the more traditional approach based on calculating the global eigenstates of the system: It relates the optical response directly to the motion of charges in the system, it has interference effects built in naturally and therefore shows the correct scalings with system size (size consistency), and it provides a Green's function expression for the response which is numerically feasiable even for very large aggregates.

The third-order response of aggregates made of two-level molecules $\left(\kappa_{n}=0\right)$ was calculated in ref 32 and was recently extended to the three-level case. ${ }^{23}$ The calculation of the expectation value of eq 2.5 requires the solution of the 
Heisenberg equations of motion for the one-exciton variables $\left\langle B_{n}\right\rangle$. To third order in the external field one obtains a set of equations in which the dynamics of $\left\langle B_{n}\right\rangle$ is coupled to higher order exciton variables. ${ }^{34}$ If we restrict ourselves to low temperatures where the nuclear motions which cause pure dephasing are frozen out, the following factorization for normal ordered products of exciton operators is applicable: $\left\langle B_{k}^{\dagger} B_{l}^{\dagger} \ldots B_{n} B_{m}\right\rangle=\left\langle B_{k}^{\dagger} B_{l}^{\dagger} \ldots\right\rangle\left\langle\ldots B_{n} B_{m}\right\rangle .{ }^{34}$ Hence the third-order polarization can be determined from the coupled dynamics of one-exciton, $\left\langle B_{n}\right\rangle$, and two-exciton variables, $\left\langle B_{m} B_{n}\right\rangle$.

We shall consider a three-pulse setup in which pulse 1 is centered at $t=0$ whereas pulses 2 and 3 are simultaneous and centered at time $\tau$

$E(\vec{r}, t)=E_{1}(t) \mathrm{e}^{i \vec{k}_{1} \vec{r}}+E_{2}(t-\tau) \mathrm{e}^{i \vec{k}_{2} \vec{r}}+E_{3}(t-\tau) \mathrm{e}^{i \vec{k}_{3} \vec{r}}+$ c.c.

with $E_{j}(t-\tau)=\left(2 \pi \delta_{j}^{2}\right)^{-1 / 2} \exp \left\{-(t-\tau)^{2} / 2 \delta_{j}^{2}\right\} \exp \left\{-i \omega_{j} t\right\}$. The delay time $\tau$ can be either positive or negative. To distinguish between the different directions of the signal in the equations of motion, we need to keep track of the phase of the optical polarization. To that end, we expand all dynamical variables as $^{35}$

$X=\sum_{\alpha_{1}, \alpha_{2}, \alpha_{3}=-\infty}^{\infty} X^{\left(\alpha_{1}, \alpha_{2}, \alpha_{3}\right)} \exp \left\{i\left(\alpha_{1} \vec{k}_{1}+\alpha_{2} \vec{k}_{2}+\alpha_{3} \vec{k}_{3}\right) \vec{r}\right\}$

To calculate the signal in the direction $\vec{k}_{s}=\vec{k}_{3}+\vec{k}_{2}-\vec{k}_{1}$ we need to solve the following set of coupled first-order differential equations $^{23}$

$$
\begin{gathered}
i \hbar \frac{\mathrm{d}}{\mathrm{d} t}\left\langle B_{m}\right\rangle^{(1,0,0)}=\sum_{n} F_{m n}\left\langle B_{n}\right\rangle^{(1,0,0)}-\mu_{m} E_{1}(t) \\
i \hbar \frac{\mathrm{d}}{\mathrm{d} t}\left\langle B_{m} B_{n}\right\rangle^{(0,1,1)}=\left(1-\delta_{m n}\right)\left\{\sum _ { l } \left[F_{m l}\left\langle B_{l} B_{n}\right\rangle^{(0,1,1)}+\right.\right. \\
\left.F_{n l}\left\langle B_{m} B_{l}\right\rangle^{(0,1,1)}\right]-\left[E_{2}(t-\tau)\left[\mu_{n}\left\langle B_{m}\right\rangle^{(0,0,1)}+\mu_{m}\left\langle B_{n}\right\rangle^{(0,0,1)}\right]+\right. \\
\left.\left.E_{3}(t-\tau)\left[\mu_{n}\left\langle B_{m}\right\rangle^{(0,1,0)}+\mu_{m}\left\langle B_{n}\right\rangle^{(0,1,0)}\right]\right]\right\}+\delta_{m n}\left\{\left(2 \hbar \Omega_{m}+\right.\right. \\
\left.\Delta_{m}-i \hbar \Gamma_{m}^{(2)}\right)\left\langle B_{m} B_{m}\right\rangle^{(0,1,1)}-\kappa_{m}{ }^{2}\left[\mu_{m} E_{2}(t-\tau)\left\langle B_{m}\right\rangle^{(0,0,1)}+\right. \\
\left.\left.\mu_{m} E_{3}(t-\tau)\left\langle B_{m}\right\rangle^{(0,1,0)}-\sum_{l} J_{m l}\left\langle B_{l} B_{m}\right\rangle^{(0,1,1)}\right]\right\}
\end{gathered}
$$

and

$$
\begin{gathered}
i \hbar \frac{\mathrm{d}}{\mathrm{d} t}\left\langle B_{m}\right\rangle^{(-1,1,1)}=\sum_{n} F_{m n}\left\langle B_{n}\right\rangle^{(-1,1,1)}+ \\
\Delta_{m}\left\langle B_{m}^{\dagger}\right\rangle^{(-1,0,0)}\left\langle B_{m} B_{m}\right\rangle^{(0,1,1)}+\left(\kappa_{m}^{2}-2\right)\left\langle B_{m}^{\dagger}\right\rangle^{(-1,0,0)} \times \\
{\left[\sum_{n} J_{m n}\left\langle B_{n} B_{m}\right\rangle^{(0,1,1)}-\mu_{m}\left[E_{2}(t-\tau)\left\langle B_{m}\right\rangle^{(0,0,1)}+\right.\right.} \\
\left.\left.E_{3}(t-\tau)\left\langle B_{m}\right\rangle^{(0,1,0)}\right]\right]
\end{gathered}
$$

The equations for $\left\langle B_{m}\right\rangle^{(0,1,0)}$ and $\left\langle B_{m}\right\rangle^{(0,0,1)}$ are identical to eq 2.8 with $E_{1}(t)$ replaced by $E_{2}(t-\tau)$ or $E_{3}(t-\tau)$, respectively. Note that $\left\langle B_{n}^{\dagger}\right\rangle^{(1,0,0)}=\left[\left\langle B_{n}\right\rangle^{(-1,0,0)}\right]^{*}$. In eqs $2.8-2.10$ we used the notation $F_{m n}=\delta_{m n} \hbar\left[\Omega_{m}-i \Gamma_{m}^{(1)}\right]+J_{m n}, \Gamma_{n}^{(i)}$ being a phenomenological lifetime for state $|i\rangle$ of the $n$th monomer.

The system of eqs $2.8-2.10$ has been solved using standard fourth-order Runge-Kutta method. ${ }^{36}$ From the solution of the equations of motion we calculate the $\vec{k}_{s}$ component of the polarization

$$
P_{s}(t)=\sum_{n} \mu_{n}\left\langle B_{n}\right\rangle^{(-1,1,1)}(t)+\text { c.c. }
$$

The time-resolved FWM signal is then given by

$$
S(t) \equiv\left|P_{s}(t)\right|^{2}
$$

Time-integrated photon echoes have been reported recently for the light-harvesting antennae. ${ }^{16}$ Time-resolved FWM has been conducted in other systems such as semiconductor quantum wells and chromophores in solution. ${ }^{37}$

The two essential parameters for the interplay between CDE and $\mathrm{MDE}$ are the anharmonicity, $\Delta_{n}$, i.e., the difference between the monomer transition energy for $|1\rangle \rightarrow|2\rangle$ and $|0\rangle \rightarrow|1\rangle$ optical transitions, and the ratio between the respective transition dipole matrix elements, $\kappa_{n}$. Note that according to eq $2.2 \kappa_{n}{ }^{2}$ -2 represents non-boson exciton statistics. Equations 2.9 and 2.10 clearly show that the same parameters also control all optical nonlinearities in the present model. Setting $\Delta_{n}=0$ and $\kappa_{n}=\sqrt{2}$, the system becomes a set of linearly driven harmonic oscillators; the optical response is then purely linear, all sources in eq 2.10 vanish, and $\left\langle B_{m}\right\rangle^{(-1,1,1)}=0$ at all times.

We note two important limits: First, for $\kappa_{n}=0$ the aggregate consists of two-level molecules. The operators $B_{n}^{\dagger}$ and $B_{n}$ obey Pauli commutation relations (see eq 2.2) and $\left\langle B_{n} B_{n}\right\rangle$ vanishes at all times (for a discussion of this limit see ref 34). Second, for $\kappa_{n}^{2}=2$ the exciton operators follow Bose commutation relations, and only the term proportional to $\Delta_{n}$ in eq 2.8 gives rise to a nonlinear response. The on-site variables, $\left\langle B_{n} B_{n}\right\rangle$, are then coupled via $\kappa_{m} J_{m n}$ to the two-exciton variables $\left\langle B_{m} B_{n}\right\rangle$ (first two and last term on the rhs of eq 2.9).

In sections III and IV we present calculations of time-resolved FWM techniques which are particularly suitable for probing intermolecular coherence and two-exciton dynamics. These numerical simulations are aimed at illustrating the roles of the two different types of two-photon resonances in our model of the LHC-II and the LH2. So far, the parameters $\Delta_{n}$ and $\kappa_{n}$ have only been determined experimentally for monomeric chlorophyll in solution. The protein environment in the antenna can be expected to modify the optical properties of the pigments. Therefore, we will use $\Delta_{n}$ and $\kappa_{n}$ as parameters which are tuned within limits, reasonable from the monomeric behavior. For LH2 a monomeric (BChl $a$ ) anharmonicity of $100 \mathrm{~cm}^{-15}$ and values for $\kappa_{n}$ ranging from 0.5 to 1.4 have been used previously to model nonlinear absorption spectra of the B850 band. ${ }^{38}$ The excited singlet-state absorption of Chl $a$ contained in LHC-II shows in pyridine solution a broad band around the ground state absorption maximum at $670 \mathrm{~nm},{ }^{25}$ suggesting $\Delta_{n}$ ranging from about -200 to about $200 \mathrm{~cm}^{-1}$. Depending on the wavelength, $\kappa_{n}$ varies between 0.25 and 2.5. ${ }^{25}$ For simplicity we will use equal values for $\kappa_{n}$ and $\Delta_{n}$ for all monomers of the aggregate in our simulations.

\section{Two-Pulse FWM Spectroscopy with Negative Delay}

We first consider a two-pulse FWM technique (i.e., pulse 2 and 3 are identical, $\left.\vec{k}_{2}=\vec{k}_{3}\right)$ and calculate the time-resolved signal $S(t)$ in the direction $\vec{k}_{s}=2 \vec{k}_{2}-\vec{k}_{1}$. In Figure 2 we show the relevant Feynman diagrams for positive and negative delays. If the delay $\tau$ is positive (pulse 1 comes first) this represents the two-pulse echo. When the frequencies are tuned within the one-exciton band, the signal provides information about oneexciton dynamics, which will not be considered here. For negative delay the picture is very different; it can be easily verified that for an isolated two-level system it is impossible to 

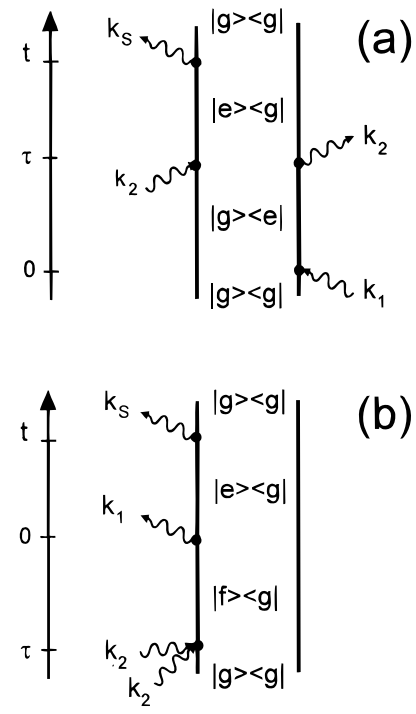

(b)

Figure 2. Double-sided Feynman diagrams representing the contributions to the FWM signal at $\vec{k}_{s}=2 k_{2}-\vec{k}_{1}$ in coupled three-level molecules which survive the rotating wave approximation: (a) ordinary photon echo (positive delay); (b) negative delay signal. In these diagrams $|g\rangle$ represents the ground state, $|e\rangle$ is the one-exciton manifold, and $|f\rangle$ is the two-exciton manifold (including both CDE and MDE).

have an all resonant process, and the signal therefore is neglegible (in the rotating wave approximation it is zero). Once a two-exciton band becomes accessible (whether MDE or CDE), a resonant signal can be generated. This setup has been shown to provide valuable information about the interaction-induced two-exciton states in coupled two-level systems. ${ }^{39,40}$

First, we consider the LH2 system. To obtain a more realistic model, we included the effect of static diagonal disorder in our simulations. To that end we assume that the monomer energies $\Omega_{n}$ have static Gaussian fluctuations. The fluctuations of various sites are independent, and all fluctuations have the same variance $\sigma_{n}$. Note that for the convoluted spectrum given in Figure 1a we assumed an inhomogeneous distribution for the entire aggregate and did not include disorder explicitly. Only for $\sigma_{n}=200 \mathrm{~cm}^{-1}$ the $\mathrm{B} 850$ bandwidth is comparable to Figure 1a. However, effects such as vibronic sidebands, for instance, are known to contribute to the B850 absorption bandwidth. Since they are not accounted for by the present approach, the correct choice of $\sigma_{n}$ is not obvious from the linear absorption profile, and $\sigma_{n}$ will be treated as a parameter subsequently. The anharmonicity is fixed at $100 \mathrm{~cm}^{-1,5}$ and $\kappa_{n}$ and $\sigma_{n}$ were varied to assume several typical values. All calculations are given for a fixed delay $\tau=-100 \mathrm{fs}$. We further took $\omega_{1}=\omega_{2}$ to be resonant with the maximum of the low-energy band (B850) which is at about $869 \mathrm{~nm}$ (see Figure 1a). Under these conditions two-exciton variables, $\left\langle B_{m} B_{n}\right\rangle$, belonging to the $\mathrm{B} 800$ ring as well as those involving both $\mathrm{B} 800$ and $\mathrm{B} 850$ pigments do not contribute significantly to the signal. We can thus reduce the numerical effort by neglecting the B800 pigments altogether in the following. The resulting time-resolved signal $S(t)$ is displayed in Figure 3.

As discussed in the Introduction, MDEs are likely to influence the exciton dynamics in LH2. For the assumed anharmonicity $\left(\Delta_{n}=100 \mathrm{~cm}^{-1}\right)$, the interaction between MDEs and CDEs causes a strong mixing between both types of two-exciton states as can be seen using eq 2.9. In the highly symmetric LH2 this interaction was shown to lead to an accumulation of oscillator strength for two-photon resonances in the two-exciton band edges upon increasing the parameter $\kappa_{n} \cdot{ }^{23}$ The prediction of this cooperative behavior was based on a model which did not include inhomogeneous broadening. Thus the question arises

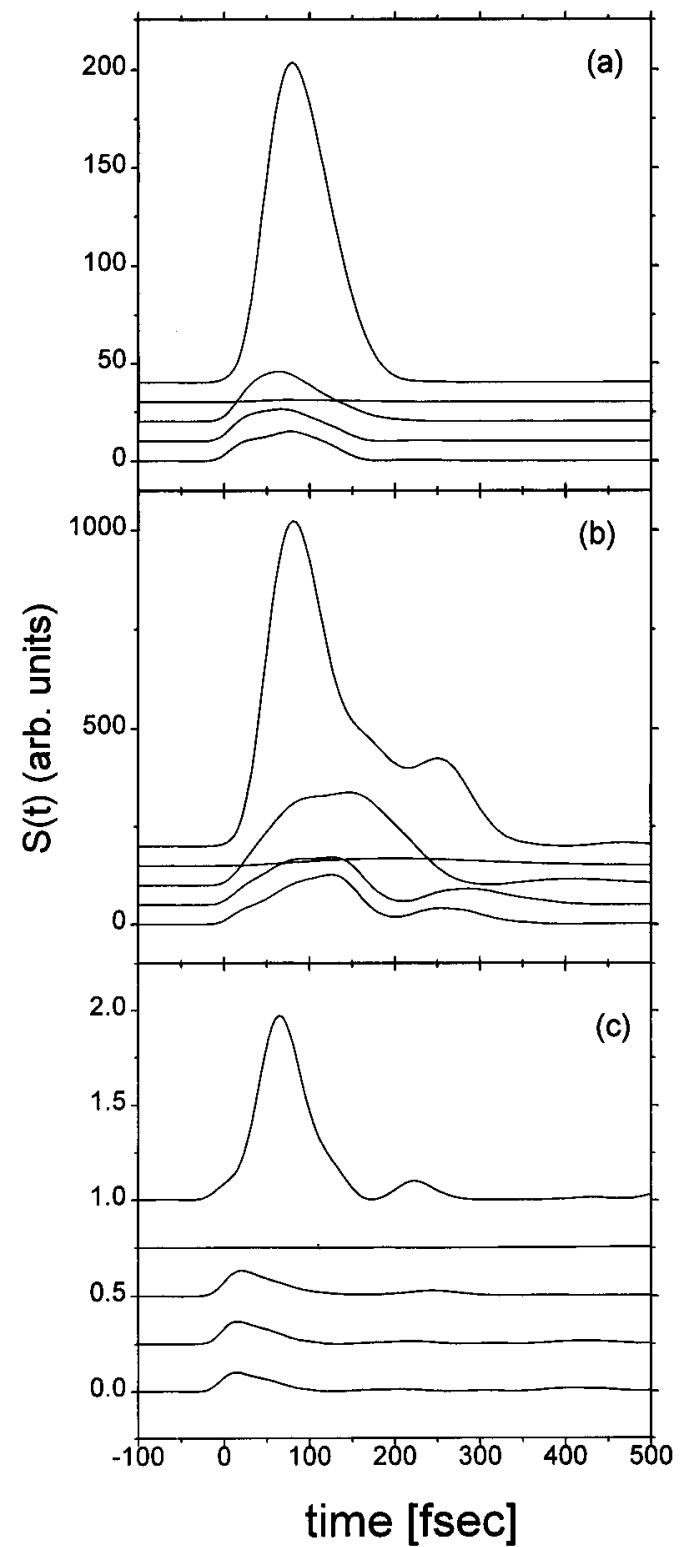

Figure 3. Time-resolved FWM signal for the B850 subsystem using pulses resonant with the low-energy band (B850) at $869 \mathrm{~nm}$ with $\delta_{j=1,2,3}$ $=17 \mathrm{fs}(40 \mathrm{fs} f w h m)$ and delay time $\tau=-100 \mathrm{fs}$ (time zero is chosen with respect to pulse 1). The variance of the static distribution of monomer energies is $\sigma_{n}=100 \mathrm{~cm}^{-1}$ (a), $50 \mathrm{~cm}^{-1}$ (b), and $200 \mathrm{~cm}^{-1}$ (c) $\left(\Gamma_{n}^{(i)}=5 \mathrm{~cm}^{-1}\right)$. In each panel we used the parameters $\kappa_{n}=0,0.5$, $1,1.5,2$ (from bottom to top) and $\Delta_{n}=100 \mathrm{~cm}^{-1}$.

whether it persists in the presence of static disorder. The signals, $S(t)$, in Figure 3 clearly show a dramatic increase for the maximum $\kappa_{n}$, i.e., $\kappa_{n}=2$, independent of the strength of disorder. As mentioned above, interference between CDEs and MDEs leads to a vanishing third-order signal in the harmonic oscillator limit, i.e., for $\kappa_{n}=\sqrt{2}$ and $\Delta_{n}=0$. In the vicinity of this limit $\left(\kappa_{n}=1.5\right.$ and $\Delta n=100 \mathrm{~cm}^{-1}$ in Figure 3$)$ the signal is still quite weak and cannot be resolved on the present scale.

To illustrate the relative MDE or CDE characteristics of the signal we have calculated the quantity

$$
C_{m n}=(1 / T) \int_{0}^{T} \mathrm{~d} t\left|\left\langle B_{m} B_{n}\right\rangle(t)\right|
$$

which gives the average amplitude of the variable $\left\langle B_{m} B_{n}\right\rangle$ in the time interval [0:T]. This interval is chosen such that $\mid\left\langle B_{m} B_{n}\right\rangle-$ $(t) \mid$ has decayed at $t=T$ (typically $T<1 \mathrm{ps).} C_{m n}$ further provides a clear real-space picture of the signal. The amplitudes 
(a)

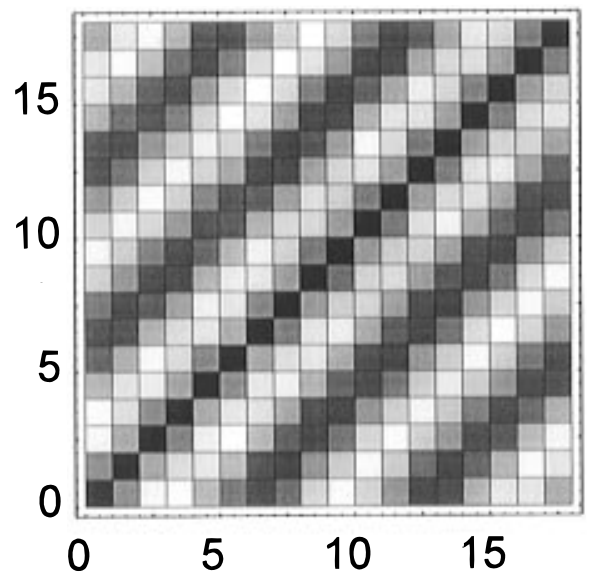

(b)

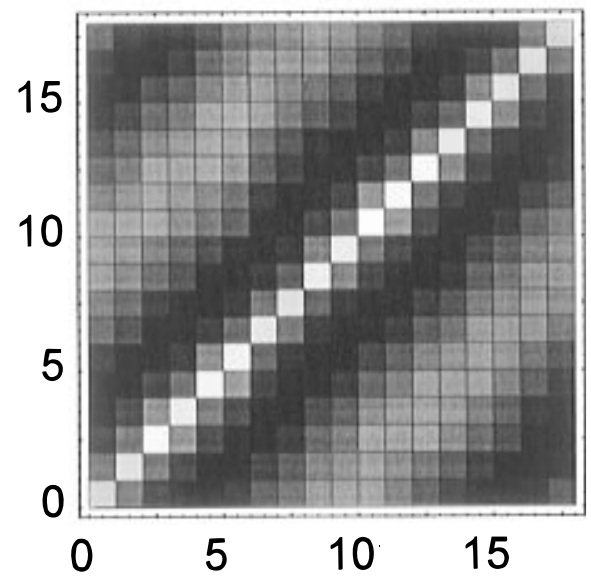

Figure 4. Function $C_{m n}$ (eq 3.1) calculated using the parameters of Figure $3 \mathrm{a}$ (a) $\kappa_{n}=0$, and (b) $\kappa_{n}=2$ (lighter shading corresponding to a higher amplitude).

$C_{m n}$ corresponding to $\kappa_{n}=0$ and $\kappa_{n}=2$ of Figure 3 a are shown in Figure 4, a and b, respectively (lighter shading corresponds to higher amplitude). In the two-level limit (Figure 4a), Pauli exclusion prevents the two excitations from occupying the same site. Therefore, the diagonal elements $C_{n n}$ are identically zero. The amplitudes of the off-diagonal elements reflect the contributions of the different molecular states to the two-exciton eigenstates for given pulse parameters. Obviously, in the present case, where excitation is at the maximum of the B850 absorption band, two-exciton states of rather high symmetry dominate the signal. The calculated signal for $\kappa_{n}=2$ (Figure 3a, uppermost curve) is dominated by MDEs. This can be seen from $C_{m n}$ plotted in Figure 4b: The diagonal elements (MDEs) have the largest amplitude, but off-diagonal elements (CDEs) still give a substantial contribution.

In Figure 3, parts b and c, we show how $S(t)$ behaves for different strengths of disorder $\left(\sigma_{n}=50 \mathrm{~cm}^{-1}(\mathrm{~b}), 200 \mathrm{~cm}^{-1}\right.$ (c)). Comparing the two panels we first note that upon increase $\sigma_{n}$ from 50 to $200 \mathrm{~cm}^{-1}$ the signal's amplitude decreases by almost 3 orders of magnitude. This is to be expected, since the signal builds up on coherences between CDEs and MDEs. However, the increase of the maximum amplitude for $\kappa_{n}=2$ is observed even for $\sigma_{n}=200 \mathrm{~cm}^{-1}$ (Figure 3c). Finally, we note that especially for weak disorder (Figure 3b), well pronounced quantum beats due to simultaneous excitation of several twoexciton states can be observed.

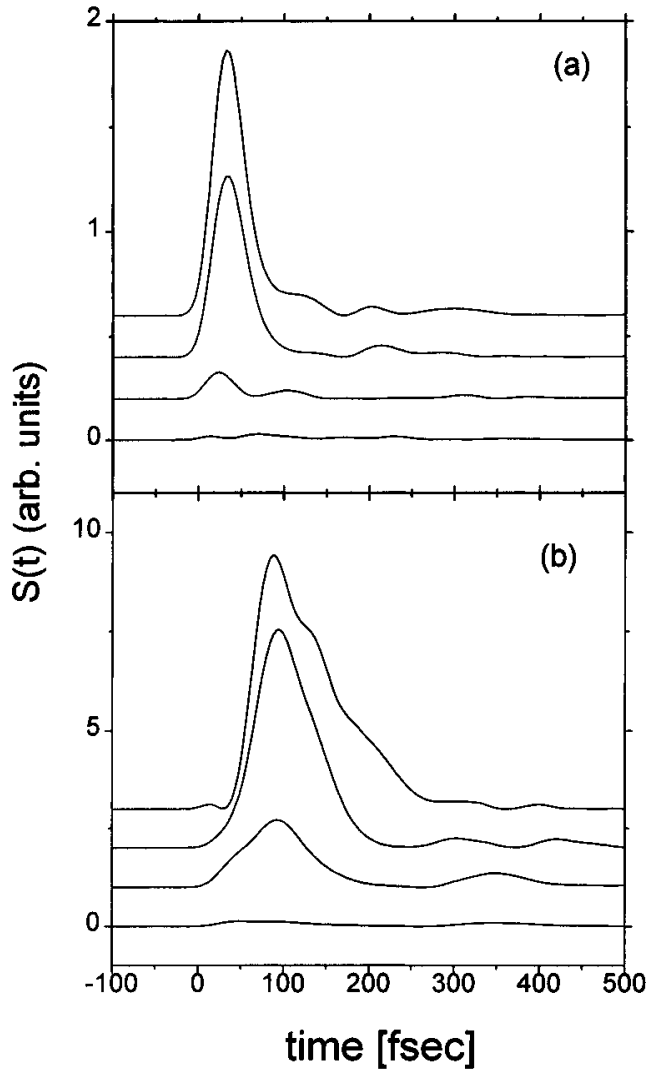

Figure 5. Time-resolved FWM signal for LHC-II calculated using pulses resonant with the major peak of the low-energy band at $676 \mathrm{~nm}$ with $\delta_{j=1,2,3}=17 \mathrm{fs}$ ( $40 \mathrm{fs}$ fwhm) and delay time $\tau=-100 \mathrm{fs}$ (time zero is chosen with respect to pulse 1 ). The variance of the static distribution of monomer energies is $\sigma_{n}=64 \mathrm{~cm}^{-1}$ and $\Gamma_{n}^{(i)}=5 \mathrm{~cm}^{-1}$ $\left(\Delta_{n}=200 \mathrm{~cm}^{-1}\right.$ (a), $\Delta_{n}=-200 \mathrm{~cm}^{-1}$ (b)).

We next turn to the LHC-II system of green plants. Here the excitation frequencies are chosen to be resonant with the strongest absorption peak of the Chl $a$ dominated band, which is centered at about $676 \mathrm{~nm}$ (see Figure 1b). Thus we probe the dynamics at the lower band edge of the two-exciton manifold as in the LH2 system. Concerning the static disorder we assume that the considerable heterogeneity of the energy level structure is accounted for by choosing distinct monomer transition energies for the different pigments. (see caption to Figure 1). The spectra are thus expected to be much less sensitive to energetic disorder: we therefore do not take into account independent fluctuations as in the $\mathrm{LH} 2$ and simply included the line broadening as shown in the dash-dotted curve in Figure $1 \mathrm{~b}$.

In Figure 5 we display $S(t)$ for $\Delta_{n}=200 \mathrm{~cm}^{-1}$ (a) and $\Delta_{n}=$ $-200 \mathrm{~cm}^{-1}$ (b) $\left(\sigma_{n}=64 \mathrm{~cm}^{-1}\right)$ and their dependence on the parameter $\kappa_{n}$. Compared to LH2 we do not observe a dramatic increase of the maximum amplitude of $S(t)$ for $\kappa_{n}=2$. This is in accord with our previous observation that the nonsymmetric structure of the LHC-II does not support the cooperative behavior found in LH2 for reasonable $\kappa_{n}{ }^{23} \mathrm{We}$ further note that there is no significant decrease of the signal close to the harmonic oscillator value $\kappa_{n}=\sqrt{2}$ since the anharmonicity is rather strong here. In contrast to LH2, both pigment pools, i.e., $\mathrm{Chl} a$ and $\mathrm{Chl} b$, contribute to the signal as can be seen from the amplitudes $C_{m n}$ plotted in Figure 6. Figure 6a shows the two-level limit; i.e., the diagonal elements $C_{n n}$ are again equal to zero. The largest amplitudes found for $n=$ 1-7 correspond to the $\mathrm{Chl} a$ molecules which are mainly excited at $676 \mathrm{~nm}$. However, off-diagonal elements involving $\mathrm{Chl} b 3$ $(n=10)$, which is strongly coupled to the Chl $a$ pool in the 
(a)

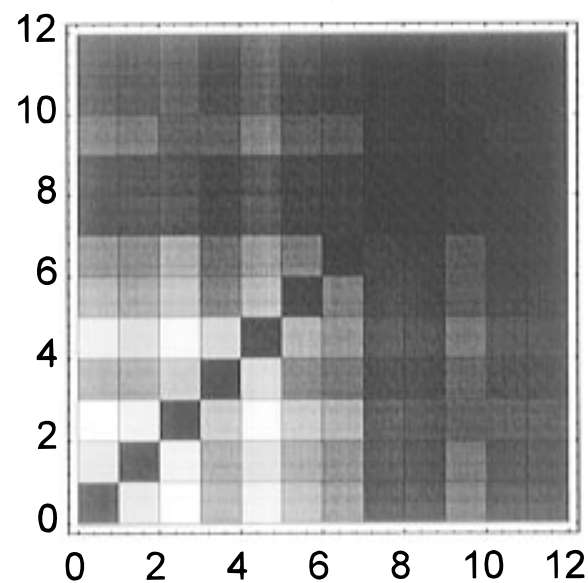

(b)

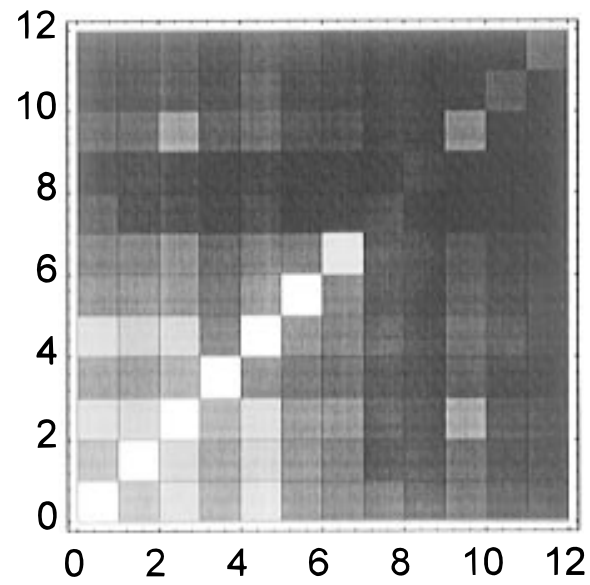

Figure 6. Same as in Figure 4 but for the LHC-II signal shown in Figure 5b.

present model, also carry appreciable amplitude. Including MDEs gives considerable contributions to the signal as seen in Figure $6 \mathrm{~b}$ for $\sigma_{n}=-200 \mathrm{~cm}^{-1}$ and $\kappa_{n}=2$. Moreover, MDEs belonging to the $\mathrm{Chl} b$ molecules with the lowest transition energies (Chl b3, b5, b6, i.e., $n=10,11,12$ ) become increasingly important for broad-band excitation at $676 \mathrm{~nm}$.

Finally, comparing panels (a) and (b) of Figure 5 we note the higher amplitudes as well as the more pronounced quantum beats for negative $\Delta_{n}$. The reason is that for $\Delta_{n}>0$, monomeric MDEs belonging to $\mathrm{Chl} b$ and some Chl $a$ molecules are shifted to higher energies and no longer contribute to the two-exciton states probed with $676 \mathrm{~nm}$ excitation.

\section{Two-Exciton Photon Echoes}

So far we considered the FWM signal for negative delay times, which is solely induced by two-exciton transitions (whether intermolecular or intramolecular). As indicated earlier, the ordinary photon echo (Figure $2 \mathrm{a}$ ) probes the dynamics of one-exciton states. However, a three-color photon echo sequence can be employed to specifically probe two-exciton dynamics. ${ }^{33}$ The pulse sequence is shown in Figure 7a. Pulse 1 comes first and is tuned on resonance with the one-exciton band. Pulses 2 and 3 follow after a delay time $\tau$ with frequencies tuned off-resonant from the one-exciton band, but with $\omega_{2}+\omega_{3}$ inside the two-exciton band. The signal is observed at $\vec{k}_{s}=\vec{k}_{3}+\vec{k}_{2}-\vec{k}_{1}$. The origin of this echo can be traced by examining the time evolution of the bra and ket vectors (a)

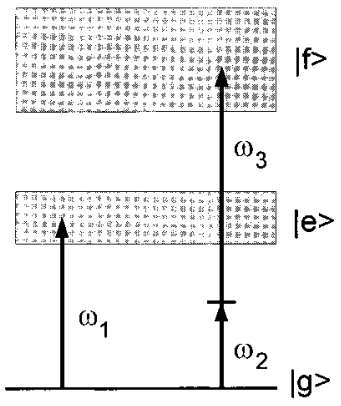

(b)

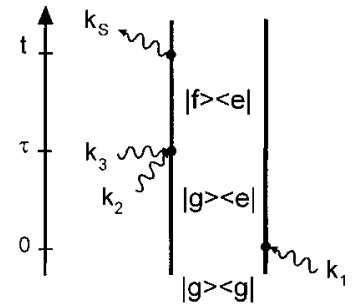

Figure 7. Pulse setup for the two-exciton photon echo at $\vec{k}_{s}=\vec{k}_{3}+\vec{k}_{2}$ $-k_{1}$ : (a) level scheme ( $|g\rangle$ ground state, $|e\rangle$ single exciton state, $|f\rangle$ two-exciton state), (b) the relevant double-sided Feynman diagram in rotating wave approximation.

in Liouville space shown in Figure 7b: Starting in the ground state $|g\rangle\langle g|$, pulse 1 (resonant with a one-exciton state, $|e\rangle$ ) prepares a coherence $|g\rangle\langle e|$ at time $t=0$. At $t=\tau$ pulses 2 and 3 (resonant with a two-exciton state, $|f\rangle$ ) switch the system into the coherence $|f\rangle\langle e|$. In the presence of dephasing due to static energetic disorder, this leads to an echo signal at $t=2 \tau$ provided the static fluctuations in $\omega_{e g}$ and in $\omega_{f e}$ are correlated.

In Figure 8 we display the third-order signal for the B850 ring in the two-exciton photon echo setup ( $\tau=250 \mathrm{fs}$ ) for two values of static disorders: $\sigma_{n}=100 \mathrm{~cm}^{-1}$ (panel (a)) and $\sigma_{n}=$ $200 \mathrm{~cm}^{-1}$ (panel (b)). In Figure 8a the variance of the static disorder is of the order of the spacing between adjacent oneexciton energy levels in the B850 band. No two-exciton echo is observed in this case. Increasing the strength of disorder such that $\sigma_{n}$ is about twice as large as the energy level spacing leads to a regime where the two-exciton echo clearly shows up in the signal (Figure 8b). It should be emphasized, however, that the redistribution of oscillator strength for two-photon transitions which takes place as the ratio between the transition dipole matrix elements, $\kappa_{n}$, is varied can also cause the system to become more anharmonic. This occurs if no oscillator strength is carried by two-exciton states at $\omega_{2}+\omega_{3}$. From Figure $8 \mathrm{~b}$ it is clear that for the chosen parameters, $\kappa_{n}=1-1.5$ yields a two-exciton echo at about $2 \tau=500 \mathrm{fs}$.

The heterogeneous energy level structure of LHC-II is more suitable for generating the two-exciton photon echo as can be seen in Figure 9. Again we tuned $\omega_{1}$ to the maximum of the Chl $a$ band (676 nm) but $\omega_{2}$ and $\omega_{3}$ far off-resonant from the one-exciton band. As in Figure 5 the static disorder is assumed to be fully correlated, corresponding to the Gaussian broadened spectrum in Figure 1b. For the chosen value of $\sigma_{n}=64 \mathrm{~cm}^{-1}$ a pronounced echo signal appears which vanishes with decreasing $\sigma_{n}$ as in the LH2. Similar to the B850 ring we observe that the shape and the maximum position of the echo signal depend on $\kappa_{n}$, i.e., on the energy level structure of the two-exciton manifold. The quantum beats are due to simultaneous excitation of several one- and two-exciton states. They are more pronounced as in the $\mathrm{B} 850$ ring of $\mathrm{LH} 2$ since more distinct excited transitions are excited for the given pulse parameters. Note that as in Figure $5 \mathrm{~b}$, negative values of $\Delta_{n}$ lead to increased excitation of Chl $b$ dominated two-exciton states, yielding the more pronounced beats seen in Figure $9 \mathrm{~b}$. 


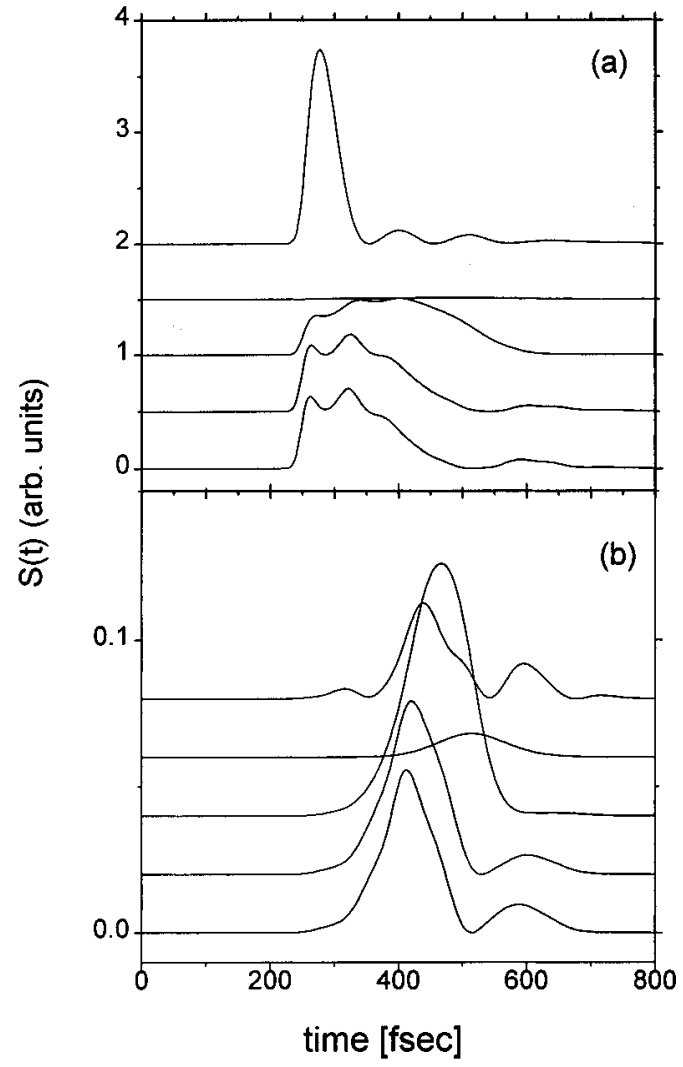

Figure 8. Time-resolved FWM signals, $S(t)$, in the B850 system for two-exciton photon-echo setup of Figure $6\left(\tau=250 \mathrm{fs}, \delta_{j=1,2,3}=17\right.$ fs). Pulse 1 is resonant with the B850 absorption maximum $(869 \mathrm{~nm})$. In each panel $\kappa_{n}=0,0.5,1,1.5,2$ (from bottom to top), $\Delta_{n}=100$ $\mathrm{cm}^{-1}$, and $\Gamma_{n}^{(i)}=5 \mathrm{~cm}^{-1}\left(\sigma_{n}=100 \mathrm{~cm}^{-1}\right.$ (a) and $200 \mathrm{~cm}^{-1}$ (b)).

\section{Summary}

The excitonic oscillator representation of the nonlinear optical response provides a powerful tool for the calculation and detailed analysis of ultrafast time-resolved FWM signals of molecular aggregates. We have applied this approach to the investigation of the two-exciton band in the structurally different lightharvesting antenna complexes of photosynthetic bacteria and green plants made of three-level chlorophyll molecules.

First, we demonstrated how the dynamics in the two-exciton band may be probed using negative delay times in the FWM setup. The signals obtained for the two antenna systems show a different dependence on the parameters governing the influence of the higher excited intramolecular state, i.e. its transition energy, $2 \hbar \Omega_{n}+\Delta_{n}$, and the respective ratio between transition dipole matrix elements, $\kappa_{n}=\mu_{n}^{(21)} / \mu_{n}^{(10)}$. In particular, the cooperative enhancement of two-photon transitions into the twoexciton manifold of LH2 predicted in ref 23 showed up as a dramatic increase of the signal upon increasing $\kappa_{n}$. We further suggested that the amplitudes of the two-exciton variables, $\left\langle B_{m} B_{n}\right\rangle$, should provide a convenient means for tracing the nature of signals stemming from two-exciton states.

The two-exciton photon echo, ${ }^{33}$ which reflects energetic disorder and strong excitonic correlations, has been calculated for both antenna complexes. It was shown that the echo in LH2 is much more sensitive to the static disorder strength and the parameters of the third intramolecular level compared to LHCII. Furthermore, the energetics in LHC-II yields well pronounced quantum beats in the echo signal, in contrast to the rather smooth behavior in $\mathrm{LH} 2$, where the oscillator strength is carried by only a few optical transitions.

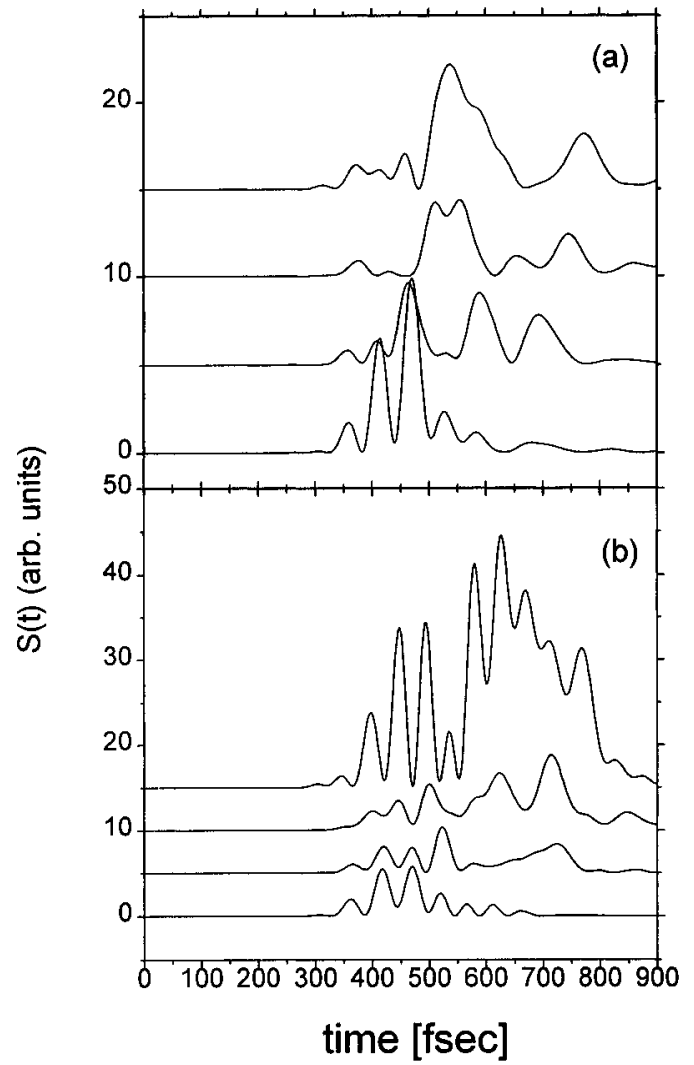

Figure 9. The time-resolved FWM signal, $S(t)$, in LHC-II for twoexciton photon-echo setup of Figure $6\left(\tau=250 \mathrm{fs}, \delta_{j=1,2,3}=17 \mathrm{fs}\right)$. Pulse 1 is tuned in resonance with the Chl $a$ dominated absorption maximum at $676 \mathrm{~nm}$. In each panel $\kappa_{n}=0,0.5,1,1.5,2$ (from bottom to top), $\sigma_{n}=64 \mathrm{~cm}^{-1}$, and $\Gamma_{n}^{(i)}=5 \mathrm{~cm}^{-1}\left(\Delta_{n}=200 \mathrm{~cm}^{-1}\right.$ (a) and $\left.-200 \mathrm{~cm}^{-1}(\mathrm{~b})\right)$.

The parameters used to describe the LH2 and the LHC-II systems reflect the current knowledge of these complexes. In particular, data on the characteristics of possible excited singletstate absorption of pigments embedded in the protein environment only recently become available (see e.g. refs 5 and 38 ). In this respect, two-exciton photon echo measurements should provide detailed information about excited singlet-state absorption and its interplay with collective double excitations.

The extension of the present equations of motion approach to include exciton population variables, $B_{n}^{\dagger} B_{m}$, is possible. These variables are relevant if pure dephasing, e.g., due to exciton-phonon interaction, is taken into account. ${ }^{22,34,41} \mathrm{Nu}-$ merical simulation of related transient absorption experiments ${ }^{11,17}$ should give additional insight into the pathways of exciton motion and provide a sensitive test for the theoretical models of LH2 and LHC-II.

Acknowledgment. The authors thank Dr. V. Chernyak for many useful discussions. This work was supported by the National Science Foundation, the Air Force Office of Scientific Research, and in part by a grant of High Performance Computing time from the DoD HPC Center CEWES. O.K. gratefully acknowledges a postdoctoral fellowship from the German Academic Exchange Service (DAAD).

\section{References and Notes}

(1) Van Grondelle, R.; Dekker, J. P.; Gillbro, T.; Sundström, V. Biochim. Biophys. Acta 1994, 1187, 1.

(2) Sundström, V.; van Grondelle, R. In Anoxygenic Photosynthetic Bacteria; Blankenship, R. E., Madiga, M. T., Baner C. E., Eds.; Kluwer Academic: Dordrecht, 1995; p 349.

(3) Pullerits, T.; Sundström, V. Acc. Chem. Res. 1996, 29, 381. 
(4) McDermott, G.; Prince, S. M.; Freer, A. A.; HawthornthwaiteLawless, A. M.; Papiz, M. Z.; Cogdell, R. J.; Isaacs, N. W. Nature 1995, 374, 517.

(5) Pullerits, T.; Chachisvilis, M.; Sundström, V. J. Phys. Chem. 1996, 100, 10787.

(6) Sauer, K.; Cogdell, R. J.; Prince, S. M.; Freer, A. A.; Isaacs, N. W.; Scheer, H. Photochem. Photobiol. 1996, 64, 564.

(7) In contrast to ref 5 we use the more common value for the dielectric constant, $\epsilon=2$ (see also discussion in ref 5).

(8) Wu, H. M.; Savikhin, S.; Reddy, N. R. S.; Jankowiak, R.; Cogdell, R. J.; Struve, W. S.; Small, G. J. J. Phys. Chem. 1996, 100, 12022.

(9) Kühlbrandt, W.; Wang, D. N.; Fujiyoshi, Y. Nature 1994, 367, 614.

(10) Gülen, D.; van Grondelle, R.; van Amerongen, H. in Photosynthesis: from Light to Biosphere; Mathis, P., Ed.; Kluwer Academic: Dordrecht, 1995; Vol. I, p 335.

(11) Visser, H. M.; Kleima, F. J.; van Stokkum, I. H. M.; van Grondelle, R.; van Amerongen, H. Chem. Phys. 1996, 210, 91.

(12) Renger, T. Theoretical Investigation of the Energy Transfer in the Light-Harvesting Complex of Photosystem II in Higher Plants. Diploma thesis, Humboldt University, Berlin, 1995; Voigt, J.; Renger, T.; Schödel, R.; Schrötter, T.; Pieper, J.; Redlin, H. Phys. Status Solidi (b) 1996, 194, 333.

(13) Renger, T.; Voigt, J.; May, V.; Kühn, O. In Femtochemistry-The Lausanne Conference; Chergui, M., Ed.; World Scientific: Singapore, 1996; p 401.

(14) Bittner, T.; Irrgang, K.-D.; Renger, G.; Wasielewski, M. R. J. Phys. Chem. 1994, 98, 11821.

(15) Hess, S.; Åkesson, E.; Cogdell, R. J.; Pullerits, T.; Sundström, V. Biophys. J. 1995, 69, 2211.

(16) Joo, T.; Jia, Y.; Yu, J.-Y.; Jonas, D. M.; Fleming, G. R. J. Phys. Chem. 1996, 100, 2399.

(17) Chachisvilis, M.; Pullerits, T.; Jones, M. R.; Hunter, C. N.; Sundström, V. Chem. Phys. Lett. 1994, 224, 345.

(18) Eads, D. D.; Castner, E. W.; Alberte, R. S.; Mets, L.; Fleming, G. R. J. Phys. Chem. 1989, 93, 8271.

(19) Du, M.; Xie, X.; Mets, L.; Fleming, G. R. J. Phys. Chem. 1994 $98,4736$.
(20) Pålsson, L. O.; Spangfort, M. D.; Gulbinas, V. G.; Gillbro, T. FEBS Lett. 1994, 339, 134.

(21) Bittner, T.; Wiederrecht, G. P.; Irrgang, K.-D.; Renger, G.; Wasielewski, M. R. Chem. Phys. 1995, 98, 311.

(22) Mukamel, S. In Molecular Nonlinear Optics; Zyss, J., Ed.; Academic: New York, 1994; p 1.

(23) Kühn, O.; Chernyak, V.; Mukamel, S. J. Chem. Phys. 1996, 105, 8586.

(24) Knoester, J.; C. Spano, F. C. Phys. Rev. Lett. 1995, 74, 2780.

(25) Shepanski, J. F.; Anderson, R. W. Chem. Phys. Lett. 1981, 78, 165.

(26) Kwa, S. L. S.; van Amerongen, H.; Lin, S.; Dekker, J. P.; van Grondelle, R.; W. S. Struve, W. S. Biochim. Biophys. Acta 1992, 1102, 202.

(27) Chernyak, V.; Mukamel, S. J. Chem. Phys. 1996, 104, 444. Meier, T.; Mukamel, S. Phys. Rev. Lett. 1996, 77, 3471.

(28) Mukamel, S.; Takahashi, A.; Wang, H. X.; Chen, C. G. Science 1995, 266, 250.

(29) Knoester, J.; Mukamel, S. Phys. Rep. 1991, 205, 1.

(30) Leegwater, J.; Mukamel, S. Phys. Rev. A 1992, 46, 452.

(31) Spano, F. C.; Mukamel, S. Phys. Rev. Lett. 1991, 66, 1197. Spano,

F. C.; Mukamel, S. J. Chem. Phys. 1991, 95, 7526.

(32) Chernyak, V.; Wang, N.; Mukamel, S. Phys. Rep. 1995, 263, 213.

(33) Chernyak, V.; Mukamel, S. Phys. Rev. Lett. 1995, 74, 4895.

(34) Mukamel, S. Principles of Nonlinear Optical Spectroscopy; Oxford: New York, 1995.

(35) Lindberg, M.; Binder, R.; Koch, S. Phys. Rev. A 1992, 45, 1865.

(36) Press, W. H.; Flannery, B. P.; Teuklofsky, S. A.; Vetterling, W. T. Numerical Recipes; Cambridge University Press: Cambridge, U.K., 1988

(37) Vöhringer, P.; Arnett, D. C.; Yang, T. S.; Scherer, N. F. Chem. Phys. Lett. 1995, 237, 387.

(38) Chachisvilis, M.; Pullerits, T.; Westerhuis, W.; Hunter, N. C.; Sundström, V. J. Phys. Chem., submitted for publication.

(39) Schmitt-Rink, S.; Mukamel, S.; Leo, K.; Shah, J.; Chemla, D. Phys. Rev. A 1991, 44, 2124.

(40) Leegwater, J. A.; Mukamel, S. J. Chem. Phys. 1994, 101, 7388

(41) Axt, V. M.; Kühn, O.; Mukamel, S. J. Lumin., in press. 